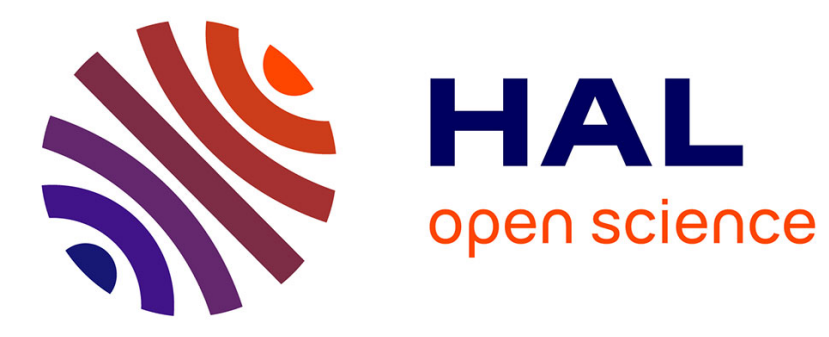

\title{
Neuromimetic Robots inspired by Insect Vision
}

Nicolas Franceschini, Stéphane Viollet, Franck Ruffier, Julien Serres

\section{To cite this version:}

Nicolas Franceschini, Stéphane Viollet, Franck Ruffier, Julien Serres. Neuromimetic Robots inspired by Insect Vision. 3rd International Conference "Smart, Materials, Structures and Systems" (CIMTEC), Jun 2008, Acireale, Sicily, Italy. hal-02296278

\section{HAL Id: hal-02296278 \\ https://hal-amu.archives-ouvertes.fr/hal-02296278}

Submitted on 25 Sep 2019

HAL is a multi-disciplinary open access archive for the deposit and dissemination of scientific research documents, whether they are published or not. The documents may come from teaching and research institutions in France or abroad, or from public or private research centers.
L'archive ouverte pluridisciplinaire $\mathbf{H A L}$, est destinée au dépôt et à la diffusion de documents scientifiques de niveau recherche, publiés ou non, émanant des établissements d'enseignement et de recherche français ou étrangers, des laboratoires publics ou privés. 


\title{
Neuromimetic Robots inspired by Insect Vision
}

\author{
Nicolas Franceschini, Stéphane Viollet, Franck Ruffier, Julien Serres \\ Institute of Movement Science (Biorobotics Lab) \\ CNRS \& Univ of the Mediterranean \\ 163 Avenue de Luminy (CP938) \\ 13288, MARSEILLE (France) \\ nicolas.franceschini@univmed.fr, stephane.viollet@univmed.fr, \\ franck.ruffier@univmed.fr, julien.serres@univmed.fr
}

\begin{abstract}
Equipped with a less-than-one-milligram brain, insects fly autonomously in complex environments without resorting to any Radars, Ladars, Sonars or GPS. The knowledge gained during the last decades on insects' sensory-motor abilities and the neuronal substrates involved provides us with a rich source of inspiration for designing tomorrow's self-guided vehicles and micro-vehicles, which are to cope with unforeseen events on the ground, in the air, under water or in space. Insects have been in the business of sensory-motor integration for several 100 millions years and can therefore teach us useful tricks for designing agile autonomous vehicles at various scales. Constructing a "biorobot" first requires exactly formulating the signal processing principles at work in the animal. It gives us, in return, a unique opportunity of checking the soundness and robustness of those principles by bringing them face to face with the real physical world. Here we describe some of the visually-guided terrestrial and aerial robots we have developed on the basis of our biological findings. These robots (Robot Fly, SCANIA, FANIA, OSCAR, OCTAVE and LORA) all react to the optic flow (i.e., the angular speed of the retinal image). Optic flow is sensed onboard the robots by miniature vision sensors called Elementary Motion Detectors (EMDs). The principle of these electro-optical velocity sensors was derived from optical/electrophysiological studies where we recorded the responses of single neurons to optical microstimulation of single photoreceptor cells in a model visual system: the fly's compound eye. Optic flow based sensors rely solely on contrast provided by reflected (or scattered) sunlight from any kind of celestial bodies in a given spectral range. These nonemissive, powerlean sensors offer potential applications to manned or unmanned aircraft. Applications can also be envisaged to spacecraft, from robotic landers and rovers to asteroid explorers or space station dockers, with interesting prospects as regards reduction in weight and consumption.
\end{abstract}

Keywords: Vision, flies, optic flow, photoreceptors, motion detection, motion detecting neurones, visuo-motor control systems, biorobotics, bionics, biomimetics

Abbreviations: $\mathrm{OF}=$ Optic Flow, EMD $=$ Elementary Motion Detector, LPTC $=$ Lobula Plate Tangential Cells 


\section{Introduction}

Animals and humans are natural «vehicles » [1], able to move about autonomously in complex environments. Insects have quite a wide behavioral repertoire and can teach us how to cope with unpredictable environments using smart sensors and limited processing resources. Insects' sensorymotor control systems are masterpieces of integrated optronics, neuronics and micromechatronics. Their neural circuits are highly complex - commensurate with the sophisticated behaviour they mediate - but unlike in vertebrates they can be investigated at the level of single, uniquely identifiable neurons, i.e., neurons that can be reliably identified in all the individuals of the species on the basis of their location in the ganglion, their exact shape and their consistent electrical responses [Rev.: 2-3]. This advantage of insect versus vertebrate neuroscience enables researchers to accumulate knowledge during anything from a few days to several decades about a given individual neuron or neural circuit. This explains why many of the robots that emulate part of a nervous system were inspired by arthropods and particularly insects [4-9].

The biologically based robots that we have been constructing since 1985 have largely contributed to creating the field of Biorobotics, in which natural principles or systems are abstracted from an animal and implemented in the form of a hardware physical model. After a short introduction to the fly visual system (Section 1), we briefly describe some of the robots we conceived and constructed (Section 2 and 3). Beyond their possible usefulness as intelligent machines, these physical models make it possible to subject biological hypotheses to the rigorous tests imposed by the real world [10-11]. Section 4 therefore deals with the "biological returns" that came out from this biorobotic approach. Section 5 deals with possible applications of these findings to aerospace.

\section{Fly neuronal microcircuits}

Flies are agile creatures that navigate swiftly through most unpredictable environments. Equipped with "only" about one million neurons and only 3000 pixels in each eye, the housefly, for example, achieves $3 \mathrm{D}$ navigation at an impressive 700 body-lengths per second. The lightness of the

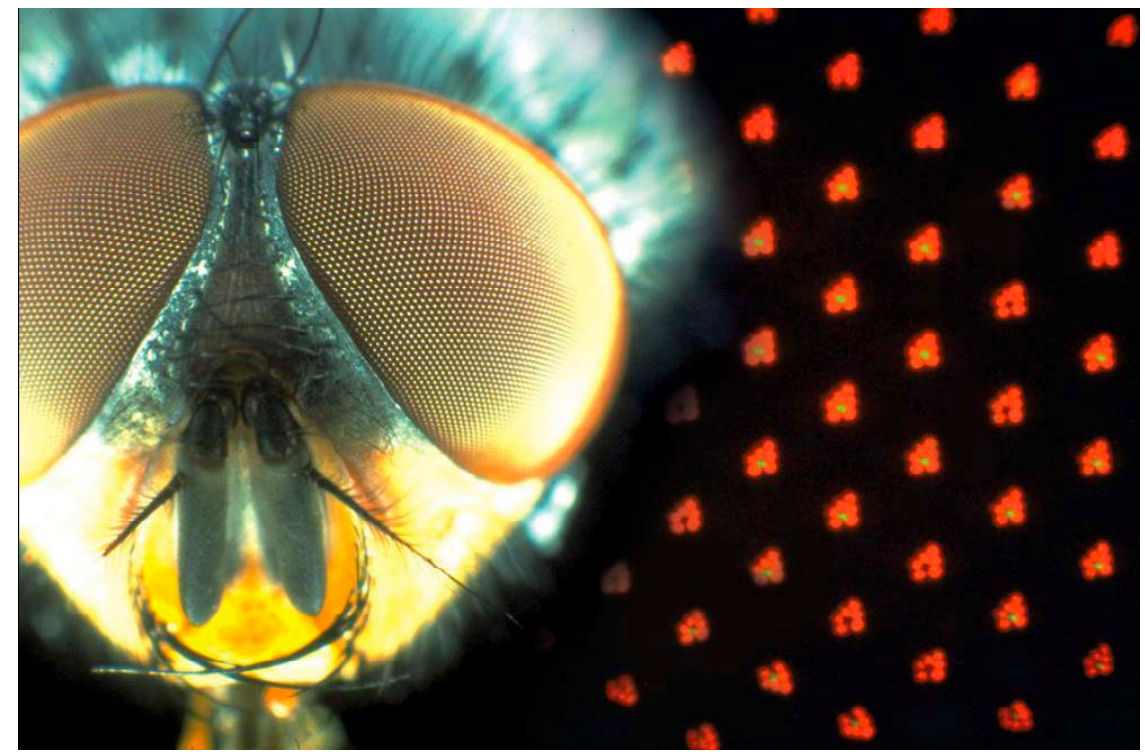

Fig.1: Head of the blowfly Calliphora erythrocephala showing the two compound eyes with their facetted cornea (left). The right part shows the retinal mosaic : behind each facet, there are 8 photoreceptor cells (each of them has a diameter of only about $1 \mu \mathrm{m}): 6$ outer receptors R1-6 and 1 central cell R7 (prolonged by an R8 cell, not seen here). These are the natural autofluorescence colors of the photoreceptors observed in vivo under strong blue excitation $[12,13]$.

Processing system at work onboard a fly makes us turn pale when we realize that these creatures actually achieve what has long been sought for in the field of autonomous robotics: dynamic stabilization, 3D collision avoidance, tracking, docking, autonomous takeoff and landing, etc. The fly compound eye consists of an array of ommatidia, each of which is covered by a facet lens (Fig.1, left) that focuses light on a small group of photorececeptor cells (Fig.1, right). 
The fly retina is among the most complex and best organized retinal mosaic in the animal kingdom. It has been described in great details, with its different spectral types of photoreceptor cells, polarization sensitive cells and sexually dimorphic cells. There exists a typical division of labour within the retina :

- The two central photoreceptor cells, R7-8, display various spectral sensitivities randomly scattered across the retinal mosaic [13-14]. They are thought to participate in color vision [15-17].

- The outer 6 photoreceptor cells (R1-R6) participate, by contrast, in motion detection [1820]. The R1-R6 photoreceptors make for a high sensitivity ("scotopic") system. In this visual pathway, signal-to-noise ratio is enhanced by the presence of an ultraviolet sensitizing pigment [74] that makes the cells "panchromatic", and by a remarkable neuronal wiring that obeys the principle of “neural superposition” [21-23].

Flying insects avoid collisions with obstacles and guide themselves through their complex surroundings by processing the optic flow (OF) that is generated on their eyes as a consequence of their locomotion. In the animal's reference frame, the OF is the angular speed $\omega$ (in $\mathrm{rad} / \mathrm{s}$ ) at which contrasting objects in the environment move past the animal [24-25]. In flies, the 3rd optic ganglion (called the Lobula Plate, LP) appears as a genuine "visual motion processing center". It comprises approximately 60 uniquely identifiable neurons, the LP tangential cells (LPTC) that analyze the OF field resulting from the animal's walking or flying, and transmit the result via the neck to thoracic interneurons.
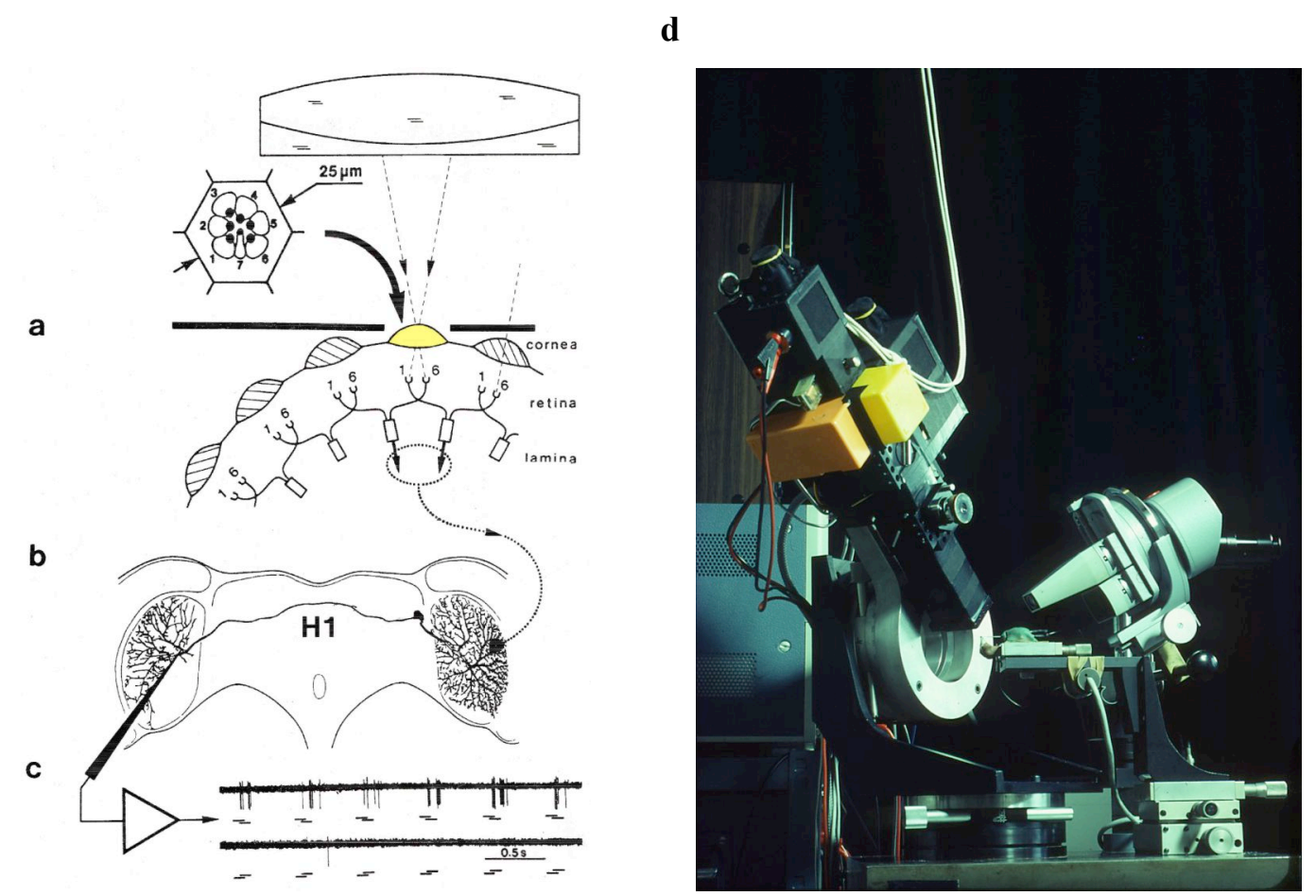

Fig. 2: (left) : (a-c) Principle of the experiment aimed at deciphering the principle of motion detection in flies, using optical stimulation of single photoreceptors. (d) Triple-beam incident light "microscope-telescope" that successively delivers a $1 \mu \mathrm{m}$ light spot to two neighboring photoreceptor cells, R1 and R6, as shown in (a). A microelectrode records the electrical response of the motion sensitive neuron H1 to this "apparent motion" [27]

These neurons in turn drive the wing-, leg-, and head-muscles [2, 28-32]. The LPTCs are large-field collator neurons that pool the input signals from many retinotopic "Elementary Motion Detectors" (EMDs) [28, 31-32]. At present, the neural circuitry underlying an EMD is still a matter of debate. Yet, taking advantage of the micro-optical techniques we had developed [Rev. 33], we were able to stimulate a single EMD in the eye of the living fly by applying optical stimuli to single identified photoreceptor cells within an ommatidium (Fig.2a), while recording the response of an identified motion sensitive neuron, called H1, in the LP [18, 26, 27]. We applied pinpoint stimulation to two 
neighboring photoreceptors (diameter $1 \mu \mathrm{m}$ ) within the selected ommatidium by means of a special stimulation instrument (Fig. 2d) whose main objective was quite simply the facet lens itself (diameter $25 \mu \mathrm{m}$, focal length $50 \mu \mathrm{m}$ ). This optical instrument [27] served to : (i) select a facet lens (Fig. 2a), (ii) select 2 of the 7 receptors (R1 and R6), and (iii) stimulate them successively with $1 \mu \mathrm{m}$-light spots. Sequential stimulation was key to producing an "apparent motion" simulating a real motion within the small visual field of the ommatidium. The H1-neuron responded by a conspicuous increase in spike rate, as long as the phase relationship between the two stimuli mimicked a movement occurring in the preferred direction (see Fig. 2c, top trace) [18, 26, 27]. From many experiments of this kind, in which carefully planned sequences of light steps and/or pulses were applied to selected receptor pairs, we established the EMD block diagram and characterized each block's dynamics and nonlinearity [26-27]. While not unveiling the cellular details of the EMD circuit, our analysis allowed the EMD principle to be understood functionally, paving the way for its transcription into another, man-made technology.

In the mid 1980's, we designed a miniature electronic EMD whose signal processing scheme approximated that of the biological EMD [34-35]. Specifically, our scheme relies on measuring the time of travel of a contrasting feature passing between two neighboring pixels. This time is further processed to give a voltage output that grows as a monotonic function of the angular velocity $\omega$ (i.e., the OF), with little influence of pattern contrast and spatial frequency. Our scheme is not a correlator scheme [36] and corresponds to the class of token-matching schemes [37]. It is therefore a true angular velocity sensor [34] that can serve as a worthy OF sensor .
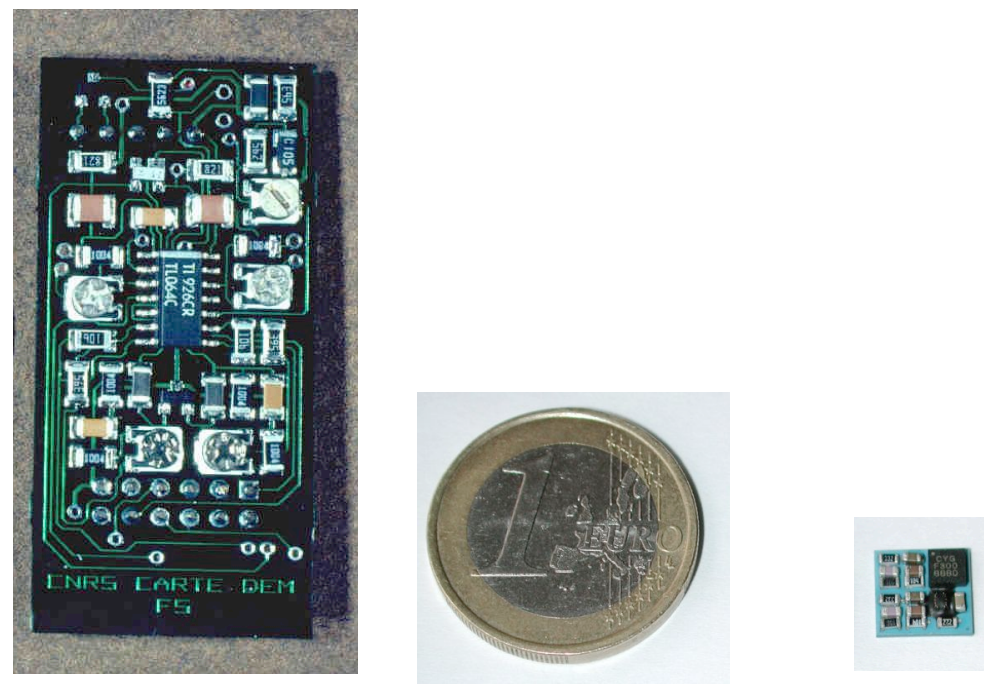

Fig.3: Opto-electronic Elementary Motion Detector (EMD) based on the functional diagram of a fly EMD established via physiological analysis (Fig.2). (a) purely analog version from 1989 (5 grams) [38]. (b) last version from 2007 based on a microcontroler and realized with low temperature co-fired ceramics (0.2 gram) [39]. Other version are based on FPGAs [40].

\section{Fly-inspired visually-guided terrestrial robots}

In the mid 1980's we designed a robot (Fig. 4a) that was to demonstrate how an agent could possibly navigate in a complex environment on the basis of optic flow. The robot was equipped with a planar compound eye and a fly-inspired EMD array that sensed the OF generated during locomotion [49]. The 50-cm-heigh "robot-mouche" (robot Fly, in English) that we realized in 1991 was the first OF-based, completely autonomous robot able to avoid obstacles on its way, while going to its target at a relatively high speed $(50 \mathrm{~cm} / \mathrm{s})[38,41-43]$.

The robot Fly was also based on ethological findings on real flies, whose most common flight trajectories consist of straight flight sequences interspersed with rapid turns termed saccades [20, 44-47]. Flight at speed V near an obstacle located at distance D and azimuth $\varphi$ with respect to the heading direction generate a translational optic flow $\omega$ expressed as follows :

$$
\omega=(\mathrm{V} \cdot \sin \varphi) / \mathrm{D}
$$


Thus, an animal (or a robot) able to measure $\omega$ can recover the distance $\mathrm{D}$ to the obstacle, provided it knows its own speed $\mathrm{V}$. The robot Fly proceeds by making purely translational steps $\Delta \mathrm{L}$ (length $10 \mathrm{~cm}$; duration $200 \mathrm{~ms}$ ) at a speed set at $\mathrm{V}=50 \mathrm{~cm} / \mathrm{s}$. By the end of each step, the panoramic EMDarray has drawn up a map of the local obstacles. The next tack to be set is immediately given, generating an "eye+body saccade" in a new direction. The robot was filmed wandering about in an arena with obstacles arranged at random. Its trajectory is very reminiscent of the jerky flight trajectories of real flies [45-48]. The robot must move in order to see. No "path planning" is required: the only thing that is "planned" is the direction of the next tack, on the basis of the OF sensed during the previous tack and the target's direction.

The robot Fly actually views the world through a horizontal ring of facets (visible at half-height in Fig.4a). Any two neighboring facets drive an EMD; a total of 114 EMDs analyze the optic flow in the azimuthal plane. The compound eye is endowed with a resolution gradient such that the interommatidial angle $\Delta \varphi$ follows the sine law of eq. 1 as a function of the eccentricity $\varphi[49,42]$. This gradient "embedded" in the optical design of the eye, makes it possible to design the electronic array uniformly, each EMD having the same time constants as its neighbors [42].

A robot of this kind was shown to automatically adjust its speed to the density of the obstacles present in the environment if, instead of imposing constant $\Delta L$ translations upon the robot as above, one imposes constant $\Delta$ t translation times at a variable speed [50]. The novel robot Fly was shown making a detour around a dense forest, automatically accelerating in zones with distant obstacles and automatically braking upon entering a "sparsely planted forest" [50].

\section{Insect-inspired visually-guided aerial robots}

Optic flow can be used to guide a flying agent, have it follow a rough terrain and land [51-52]. The latter paper [52] showed for the first time that it is possible to follow a terrain and land without measuring groundspeed and groundheight. Following simulation experiments, the principle was first validated on-board FANIA, a miniature tethered helicopter having a single variable pitch rotor, an accelerometer and a forward looking eye with a resolution of only 20 pixels [53]. This $0.8-\mathrm{kg}$ rotorcraft had only 3 degrees of freedom (forwards, upwards and pitch). Mounted at the tip of a flight mill, the robot lifted itself by increasing its rotor collective pitch. By remotely inclining the servo-vane located in the propeller wake, the operator made the helicopter pitch forward by a few degrees so that it gained speed and therefore climbed. FANIA was seen to jump over contrasting obstacles by increasing its collective pitch as a function of the fused signals transmitted from its EMDs [53].

Upon formalizing the optic flow sensed by a flying creature, we recently came up with an OF based autopilot that we call the optic flow regulator, which is little demanding in terms of neural implementation and could be just as appropriate for insects as it would be for aircraft $[54-57,11]$. We built a miniature (100-gram) helicopter, called OCTAVE, and equipped it with this autopilot (Fig.4b). Tested in its cicular arena, OCTAVE performed challenging maneuvers such as terrain following at various speeds and smooth take-off and landing [54-57, 11]. OCTAVE was also shown to react sensibly to wind perturbations [55]. In the autopilot, a ventral EMD [58] continuously measures the ventral OF and compares it to an OF set-point $\omega_{\text {set. }}$ The error signal $\varepsilon$. essentially controls the robot's lift $\mathbf{L}$ (for details see $[56,11]$ ) and hence its groundheight $\mathbf{h}$ via the surge dynamics, so as to maintain the perceived OF at a constant set-point $\omega_{\text {set }}$ (hence the name: $O F$ regulator). This will occur whatever the robot's current groundspeed $\mathbf{V}_{\mathbf{x}}$, whatever disturbances (such as wind) affect that speed, and whatever disturbances (such as a gently sloping terrain) affect the robot's current groundheight $\mathbf{h}$ : in all cases, the optic flow regulator will automatically generate a groundheight proportional to the groundspeed. Two noteworthy results were obtained in these studies:

1/ risky manœuvres such as automatic takeoff, ground avoidance, terrain following, suitable wind reactions and landing are all successfully performed as a result of the optic flow regulator.

$2 /$ the optic flow regulator does not require explicit knowledge of absolute altitude, groundheight, groundspeed, airspeed, ascent (or descent) speed and windspeed. 

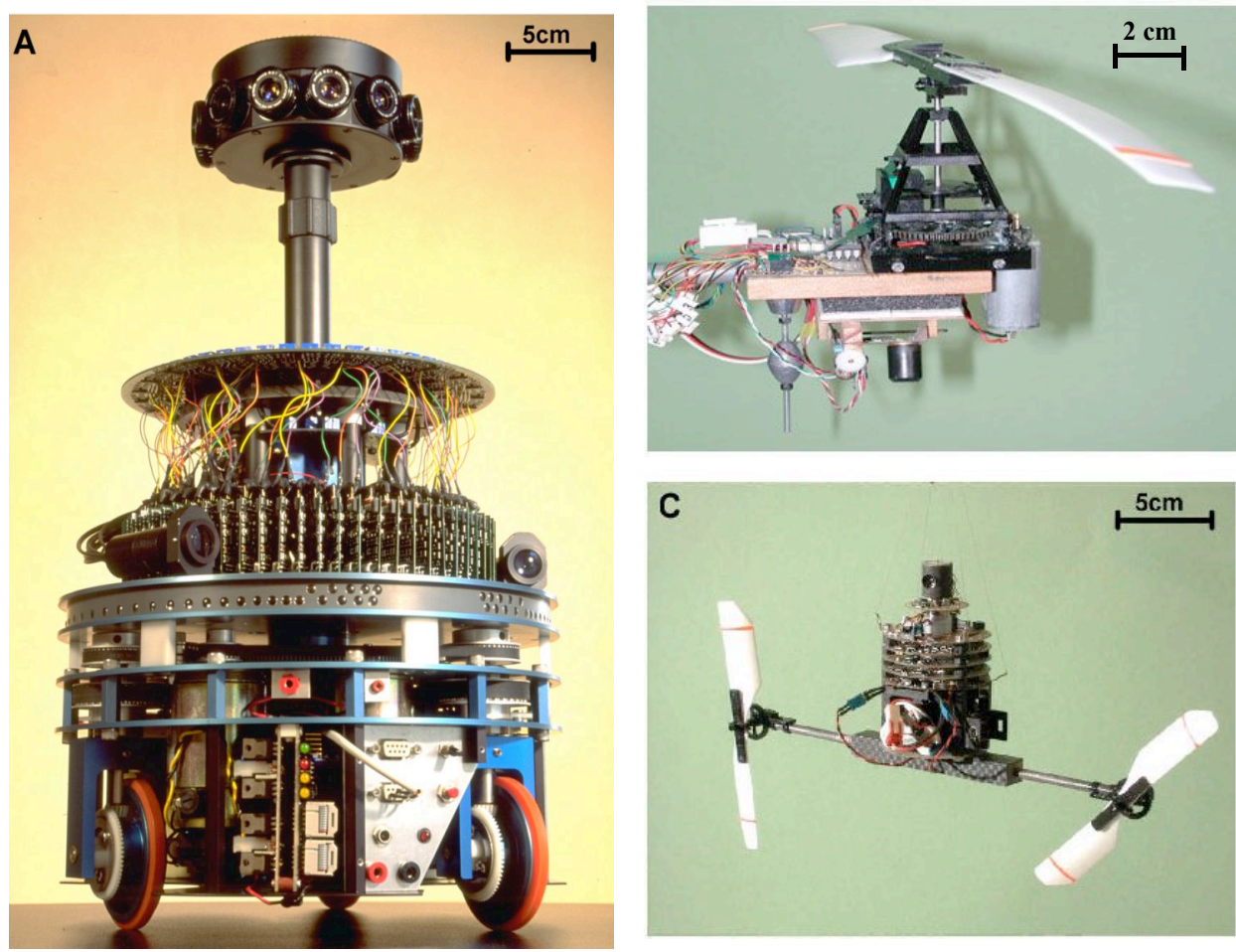

Fig.4: Three of the visually-guided robots designed and constructed at the Laboratory on the basis of our biological findings.

(a) The robot Fly aims to go to its target (detected by the dorsal eye), while avoiding obstacles (detected by the compound eye, visible at half height). The robot is fully autonomous as regards processing and power resources [42-43].

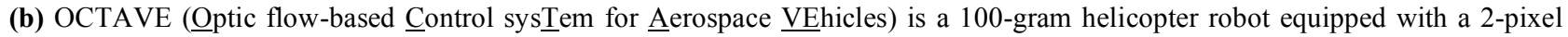
eye sensing the OF on the terrain below. Tethered to a flight mill, OCTAVE ascends or descends depending on the ventral optic flow measured [54-56]. (c) OSCAR (ㅁpptic flow based S Scanning sensor for the Control of $\underline{A}$ erial Robots) is a 100-gram, twin-engined aircraft equipped with a two-pixel scanning eye inspired by a fly ommatidium. It is tethered to a 2-meter-long nylon wire secured to the ceiling of the laboratory. OSCAR fixates and tracks a target (a dark edge or a bar) with hyperacuity [59].

This bioinspired autopilot therefore strikingly differs from conventional man-designed autopilots, which need a large number of metric sensors (e.g., a radar-altimeter, a baro-altimeter, a Doppler radar, a laser range finder, a GPS receiver, a Pitot tube, a variometer, etc.) to achieve aircraft guidance. OCTAVE autopilot's objective is not to produce altitude hold or speed hold. The objective is to constantly adapt the groundheight to the groundspeed to avoid crashing. It does so at any groundspeeds, raising the robot in proportion to its current groundspeed - whatever internal or external factors affect the latter - without ever measuring groundspeed or groundheight at all [60]. Along the same line, we recently showed that a fully actuated hovercraft can achieve both lateral obstacle avoidance and cruise control in a corridor, by means of a dual OF regulator [61]. In simulation experiments, our OF-based autopilot, called LORA III, was shown to automatically adjust both the robot's groundspeed and its clearance from the walls without any needs for measuring groundspeed and distance. In other words, the robot achieves this behaviour although it is completely 'unaware' of its own groundspeed, unaware of its clearance from the walls, and unaware of the actual corridor width. The LORA III robot navigates at sight on the sole basis of two parameters, which are the set-points of the two intertwined OF regulators: a sideways OF set-point and a forward OF set-point. These two parameters alone constrain the vehicle's behaviour fully in a straight or tapered corridor, leading either to wall-following or centering [61].

Electrophysiological recordings on flying flies with soft microelectrodes has revealed that the muscle attached to the base of the retina [62] causes retinal microscanning: the whole retinal mosaic (Fig.1a) translates repeatedly (at about $5 \mathrm{~Hz}$ ) by a few micrometers underneath the facet mosaic, causing the visual axes to rotate by a few degrees [63]. Therefore, the two novel robots we built on this basis, SCANIA and OSCAR, exploit a purely rotational optic flow. Both projects are based on the hypothesis that microscanning in flies operates in connection with motion detection.

SCANIA is a $0.7-\mathrm{kg}$ wheeled Cyclopean robot that is able to move about under visual control, avoiding the contrasting walls of a square arena despite the low resolution of its eye (only 24 
pixels) [64]. This ability is the result of a symmetrical anterograde retinal microscanning process, which assists the robot in detecting obstacles located near the heading direction (i.e., near the frontal "pole" of the optic flow field, defined as $\varphi=\mathbf{0}$ in eq.1). The periodic microscanning amounts to periodically adding a given amount of rotational optic flow $\omega_{\mathbf{r}}$ to the very small translational optic flow $\omega_{\mathbf{t}}$ generated by frontal obstacles, thereby improving the detection of small translational OF that would otherwise have remained subliminal.

The second project that we developed on the basis of the fly's retinal microscanner ended up with a novel optronic sensor [65], and a novel aerial robot, OSCAR equipped with this sensor [66, 69]. OSCAR (Fig. 4c) is attached to a thin, 2-meter long wire secured to the ceiling of the laboratory. It is free to adjust its yaw by driving its two propellers differentially (Fig. 4c). Thanks to its microscanning eye, this miniature (100-gram) robot is able to fixate a nearby "target" (a dark edge or a bar) and track it at angular speeds of up to $30 \% \mathrm{~s}$ - a value similar to the maximum tracking speed of the human eye. The OSCAR sensor was shown to be able to locate an edge with a resolution of 0.1 degrees, which is 40 times smaller than the interreceptor angle $\left(\Delta \varphi=4^{\circ}\right)$ : it has acquired hyperacuity [59]. This property makes the principle appealing for accurate stabilization of various platforms [67], as well as for nonemissive power line detectors on-board fullscale helicopters [68]. Inspired by the fly's thoracic halteres that have long been known to act as gyroscopes, we equipped the OSCAR robot with an additional rate control loop based on a MEMS rate gyro. The interplay of the two (visual and inertial) control loops enhances both the stability and the dynamic performances of the yaw fixation and pursuit system [66, 59]. More recently we introduced a mechanical decoupling between eye and body, and implemented a vestibulo-ocular reflex that was key to maintaining the robot's gaze perfectly fixed on the target [69]. Latest development gave rise to OSCAR II, a 50-gram aerial robot whose gaze, decoupled from the heading, remains locked onto a target in spite of drastic thumps that we deliberately apply to the body by means of a "slapping machine" [70].

\section{Biological returns}

Our biorobotic approach has yielded some interesting biological "returns":

- While our early terrestrial robots, the robot Fly (Fig.4a) and SCANIA, accessed the distance D to the obstacles according to eq. 1 (knowing $\boldsymbol{\theta}$ and $\mathbf{V}$ and measuring $\boldsymbol{\omega}$ with EMDs) the aerial robots FANIA, OCTAVE and LORA III are major improvements as they are able to fly around at relatively high groundspeeds without any knowledge of groundspeed and distance to the substrate, in much the same way as honeybees seem to operate [71-72, 54-56]. In a recent paper [11] we showed that the OF regulator scheme accounts for many enigmatic findings of the last decades on insects' visually guided behavior; for example, the findings that honeybees descend in a headwind, land with a constant slope and sometimes drown when flying over still water.

- The small Cyclopean robot SCANIA described in Section 3, which uses a retinal microscanner emulating the one we recently discovered in the fly compound eye, is able to recover the negligibly small translational OF associated with objects oriented close to the vehicle's heading direction. With hindsight, this suggests that the fly may also use its retinal microscanner for the same task, eliminating the problem caused by the frontal pole of the optic flow field and that caused by the relatively coarse spatial sampling of the eye $\left(\Delta \varphi \approx 1\right.$ to $\left.2^{\circ}\right)$.

- The aerial robot OSCAR, which is also based on the fly's microscanner, emits behaviors which are reminiscent of both the hoverfly's ability to maintain a steady flight position while hovering near a tree in wait for passing mates or intruders [44], and the male housefly's ability to track the female smoothly during sexual pursuit. OSCAR therefore suggests that these behavioural feats may result from the presence of the microscanner in the compound eyes, which is known to come into play only during locomotion, leaving the retina perfectly stationary at rest [63].

- With a minimalistic number (two) of OF sensors, the hovercraft LORA III shows behaviours [61] that account remarkably well for the behaviour observed in bees flying along a stationary or nonstationary corridor [73], and even along a tapered corridor [71]. This suggests that a similar dual OF regulator may well be implemented onboard the bee. 


\section{Potential applications to manned and unmanned aircraft or spacecraft}

Our insect-inspired robots simply illustrate the fact that sensory-motor control principles that have already been field tested over a decent period (hundred millions of years) onboard natural creatures can also provide vehicles with some kind of autonomy. Further developments along this line may lead to alternatives to the conventional, man-made avionic sensors. An OF sensor is a small and lightweight piece of hardware that may be suitable for air and space applications. It has the unusual advantage to be sensitive to the relative speed between two bodies. This could be, for example, the relative speed between an aircraft and a landing site or between a satellite and its mothership. An OF sensor is a passive (nonemissive) sensor that requires photons and contrast. It is therefore no good under poor visibility conditions (darkness, fog, dust, rain, snow) where it would not compete with active sensors such as Flir, Laser- or Millimeter Wave Radars. But photons and contrast are available in many terrestrial and extraterrestrial worlds such as the Moon, Mars, other planets, asteroids and comets. Should contrast deteriorate in the visible range for whatever reason (e.g., a brownout caused by a dust storm), it may reappear in other portions of the electromagnetic spectrum, between UV and terahertz, and still allow OF to be measured.

Our visually guided robots all make use of OF to carry out humble tasks such as detecting, locating, avoiding or tracking environmental features. Two principles, OCTAVE and LORA III, may pave the way for automatic piloting without any needs for measuring groundspeed, distance or altitude, thereby eliminating the need for emissive (usually heavy and power hungry) aerospace sensors. In addition, all the autopilots described put little constraints on the OF sensor's range, owing to the feedback loop (the $O F$ regulator) that constantly strives to maintain the OF near the set-point.

\section{Conclusion}

The biorobotic approach we initiated in 1985 is a transdisciplinary approach which turns out to be most rewarding because it can kill two flies with one stone:

- It yields valuable feedback information in the fields of neuroethology and neurophysiology, as it urges us to look at sensory-motor control systems from a new angle, raises new biological questions and suggests new experiments to be carried out on insects for iteratively improving our understanding.

- It can be used to implement a basic principle abstracted from nature and check its soundness, robustness and scaling on a physically embodied machine in a real physical environment. This " reconstruction approach » opens the way to novel devices and machines, particularly in the field of cheap sensory-motor control systems for autonomous vehicles and micro-vehicles. Modern aerospace technology, though extremely advanced, might benefit from taking a glance at the way star pilots such as insects have coped with complex environments for some 300 millions of years. Flying insects are not only the oldest fly-by-wire aircraft, they are able to do much with little : few neurons in the brain, few pixels in the eyes. They can no doubt teach us worthy secrets in the realm of sensory-motor control, as they do in the field of advanced materials.

\section{Acknowledgements}

We are very grateful to our colleagues from the Laboratory who participated in the design of the earlier robots (C. Blanes, F. Mura, T. Netter). We thank S. Amic for help in the design of the digital EMD, D. Dray for simulation experiments with LORA III, L. Kerhuel for the design of OSCAR II. We also thank M. Boyron, F. Paganucci and Y. Luparini for their devoted assistance in electronics and micromechanics. This research was supported by CNRS (Life Sciences, Engineering Sciences, Information Science and Technology, Cognitive Science and Microsystem Programs), by ANR and by an EU contract (IST-1999-29043)

\section{References}

[1] Braitenberg V., "Vehicles", MIT Press, Cambridge, USA (1984)

[2] Strausfeld, N..J., « Atlas of an insect brain » Springer, Berlin (1976)

[3] Comer, CM, Robertson, RM « Identified nerve cells and insect behavior» Progr. Neurobiol. 63 (2001) 409-439

[4] Barlow H.B. et al. « Natural and artificial low-level seeing systems » Clarendon Press, Oxford (1993)

[5] Srinivasan, M. Venkatesh, S. "From living eyes to seeing machines" Oxford Univ. Press, Oxford (1997)

[6] Chang, C., Gaudiano P. "Biomimetic Robotics" (Special issue) Robotics and Autonomous systems, 30 (2000)

[7] Webb, B.,Consi , T. "Biorobotics" MIT Press, Cambridge, USA, (2001) 
[8] Ayers, J. et al. « Neurotechnology for biomimetic robots » MIT Press, Cambridge, USA, (2002)

[9] Guillot, A., Meyer, J.A. « Biomimetic robotics » (Special issue). Robotics and Autonomous Systems 50 (2005)

[10] Webb, B. "Robots in invertebrate neuroscience" Nature 417 (2002) 359-363

[11] Franceschini, N., Ruffier, F., Serres, J.

«A bio-inspired flying robot sheds light on insect piloting abilities » Curr. Biol. 17 (2007) 329-335

[12] Franceschini, N. et al. « Fluorescence of photoreceptor cells observed in vivo ». Science 213 (1981)1264-1267

[13] Franceschini, N.

«Sa majesté des mouches » In: Voir l'Invisible, JP.Gex (Ed.), Paris: Omnisciences (2007), pp 28-29

[14] Franceschini, N. et al. « Sexual dimorphism in a photoreceptor » Nature 291 (1981) 241-244

[15] Hardie, R. , Franceschini, N., McIntyre, P. « Electrophysiological analysis of fly retina II/ Spectral and polarisation sensitivity in R7 and R8 » J. Comp. Physiol. 133 (1979) 23-39

[16]Franceschini, N. « Chromatic organisation and sexual dimorphism of the fly retinal mosaic » in: Photoreceptors, Borsellino A. and Cervetto L. (Eds.), Plenum Publ. Corp., New-York, (1984), pp 319-350

[17] Hardie R.C. «Functional organization of the fly retina » In: Progress in Sensory Physiology 5, Ottosson D. (Ed.), Springer, Berlin, (1985)

[18] Riehle, A., Franceschini, N.

"Motion detection in flies: parametric control over ON-OFF pathways" Exp. Br. Res. 54 (1984) 390-394.

[19] Buchner E."Behavioral analysis of spatial vision in insects"

In: Photoreception and Vison in Invertebrates, Ali, M. (Ed.), Plenum, New-York,1984, pp 561-621

[20] Heisenberg, M, Wolf ,R. « Vision in Drosophila » Berlin, Springer, (1984)

[21] Braitenberg, V. (1967) « Patterns of projection in the visual system of the fly, I/Retina-Lamina projections »

Exp. Br. Res. 3, (1967) 271-298

[22] ] Kirschfeld, K. « Die Projektion der optischen Umwelt auf das Raster der Rhabdomere im Komplexauge von Musca ». Exp. Br. Res. 3, (1967) 248-270

[23] Kirschfeld, K.; Franceschini, N.

«Optische Eigenschaften der Ommatidien im Komplexauge von Musca. Biol. Cyb. 5 (1968) 47-52

[24] Gibson, J.J., « Perception of the visual world» Houghton, Mifflin, Boston (1950)

[25] Koenderink, J.J. « Optic flow » Vis. Res. 26 (1986) 161-180.

[26] Franceschini, N.

« Early processing of color and motion in a mosaic visual system » Neuroscience Res. Suppl. 2, (1985) 17-49

[27] Franceschini, N., Riehle, A., Le Nestour, A. « Directionally Selective Motion Detection by Insect Neurons », in: Facets of Vision, D.G. Stavenga \& R.C. Hardie, Eds, Chap. 17, Springer, Berlin, (1989) pp 360-390

[28] Hausen, K. « Monocular and binocular computation of motion in the lobula plate of the fly » Verh. Dtsch. Zool. Gesellsch. 74 (1981), 49-70

[29] Hausen, K., Egelhaaf, M. « Neural mechanisms of visual course control in insects » in: Facets of Vision, Stavenga, D.G., Hardie R.C. (Eds.), Springer, Berlin, Chapt. 18, (1989) pp 391-424

[30] Krapp, H, Hengstenberg, B, Hengstenberg, R. « Dendritic structure and receptive-field organisation of optic flow processing interneurons in the fly » J. Neurophysiol. 79 (1998) 1902-1917

[31] Borst, A., Haag, J. « Neural networks in the cockpit of the fly » J. Comp. Physiol. A188 (2002) 419-437

[32] Egelhaaf, M., Kern, R. « Vision in flying insects » Curr. Opinion in Neurobiology 12 (2002) 699-706

[33] Franceschini, N. « Sampling of the visual environment by the compound eye of the fly: fundamentals and applications ».

In: Photoreceptor Optics, A.W. Snyder and R. Menzel (Eds), Springer, Berlin, pp. 98-125

[34] Franceschini, N, Blanes, C., Oufar, L.

«Passive noncontact optical velocity sensor » (in French), Dossier Technique ANVAR/DVAR N 51,549, Paris (1986)

[35] Blanes, C. «Appareil visuel élémentaire pour la navigation à vue d'un robot mobile autonome » MS Thesis in Neurosciences, University of Aix-Marseille (1986)

[36] Reichardt W. "Movement perception in insects, in: Processing of optical data by organisms and by machines" Academic Press, New-York (1969) pp. 465-493

[37] Ullman, S. "Analysis of visual motion by biological and computer systems" Computer 14 (1981) 57-69

[38] Blanes, C. « Guidage visuel d'un robot mobile autonome d'inpiration bionique » PhD Thesis, National Polytechnic Institute, Grenoble (1991). Thesis work at the Neurocybernetics Res. Group, Marseille.

[39] Pudas, M. et al. «A miniature bio-inspired optic flow sensor based on low temperature co-fired ceramics » Sensors and Actuators A, 133 (2007) 88-95

[40] Aubepart, F., Franceschini, N. « Bio-inspired optic flow sensors based on FPGA: Applications to micro-air vehicles » J. Microprocessors and Microsystems 31 (2007) 408-419

[41] Franceschini, N., Pichon, J.M., Blanes, C., « Real-time visuo-motor control: from flies to robots » IEEE

Conference on Advanced Robotics (ICAR 91), Pisa, Italy (1991) pp. 931-935

[42] Franceschini, N., Pichon, J.M., Blanes, C.

«From insect vision to robot vision » Phil Trans Roy Soc Lond B 337, (1992) 283-294

[43] Franceschini, N., Pichon, J.M., Blanes, C. "Bionics of visuomotor control" in: Evolutionary robotics: from intelligent robots to artificial life, Gomi T. (Ed.), AAAI Books, Ottawa, Canada, (1997) pp 49-67

[44] Collett, T., Land, M. 
« Visual control of flight behaviour in the hoverfly Syritta Pipiens L. » J. Comp. Physiol. A99 (1975) 1-66

[45] Wagner, H., "Flight performance and visual control of flight of the free-flying housefly Musca domestica, I/II/III” Phil Trans Roy. Soc. B312 (1986) 527-600

[46] Schilstra, C., van Hateren, J.H.

"Blowfly flight and optic flow.1/ Thorax kinematics and flight dynamics" J. Exp. Biol. 202 (1999) 1481-1490.

[47] Tammero L.F., Dickinson, M."The influence of visual landscape on the free flight behavior of the fruitfly

Drosophila Melanogaster” J. Exp. Biol. 205 (2002) 327-343

[48] Dickinson, M., Tammero, L., Tarsino, M. «Sensory fusion in free-flight search behavior of fruit flies » Neurotechnology for biomimetic robots, (J. Ayers et al., Eds.) MIT Press, Cambridge, USA, (2002) pp.573-592

[49] Pichon, J.M., Blanes, C., Franceschini, N."Visual guidance of a mobile robot equipped with a network of selfmotion sensors" In: Mobile Robots IV. Proc. SPIE 1195, Bellingham, U.S.A. (1989) pp 44-53

[50] Martin, N., Franceschini, N. « Obstacle avoidance and speed control in a mobile vehicle equipped with a compound eye » In: Intelligent Vehicles, I. Masaki (Ed.), M.I.T. Press, Cambridge, U.S.A. (1994) pp 381-386

[51] Mura, F., Franceschini, N.« Visual control of altitude and speed in a flying agent» in: From Animals to Animats, D. Cliff, P. Husbands, J.A. Meyer, S.W. Wilson, (Eds.), Cambridge M.I.T. Press (1994) pp 91-99

[52] Netter, T., Franceschini, N. « Neuromorphic optical flow sensing for nap-of-the-earth flight »

in: Mobile robots XIV, SPIE Vol. 3838, Bellingham, USA (1999) pp 208-216

[53] Netter, T., Franceschini N. "A robotic aircraft that follows terrain using a neuromorphic eye" IEEE Intern. Conf. on Intelligent Robots and Systems (IROS-02), EPFL, Lausanne (2002) pp 129-134

[54] Ruffier, F., Franceschini, N., "OCTAVE: a bioinspired visuo-motor control system for the guidance of MicroAir-Vehicles" In: Bioengineered and bioinspired systems (A. Gabriel-Vasquez, D. Abbott, R. Carmona, Eds.) SPIE Vol. 5119 (2003) pp.1-12.

[55] Ruffier, F, Franceschini, N. "Visually guided micro-aerial robot: take off, terrain following, landing and wind reaction" IEEE Intern. Cong. Robotics and Automation (ICRA04), New Orleans, USA (2004) pp. 2339-2346

[56] Ruffier, F., Franceschini, N. « Optic flow regulation: the key to aircraft automatic guidance »

Robotics and Autonomous Systems 50 (2005) 177-194.

[57] Ruffier, F., Serres, J., Franceschini, N. Automatic landing and take-off at constant slope without terrestrial aids 31th European Rotorcraft Forum, (ERF05) Florence, Italie (2005) 71: 1-9.

[58] Ruffier, F. et al. "Bio-inspired optical flow circuits for the visual guidance of micro-air vehicles" IEEE Int. Symp. Circuits Systems, ISCAS 03, Bangkok, Thailand (2003) pp. 846-849

[59] Viollet, S, Franceschini, N.« Superaccurate visual control of an aerial minirobot» In: Autonomous Minirobots for research and edutainment U. Rückert et al. (Eds.), H. Nixdorf Institut, Paderborn, Germany (2001) pp 215-224

[60] Franceschini, N. et al.

« Steering aid system for altitude and horizontal speed, perpendicular to the vertical, of an aircraft and aircraft equipped therewith ». International Patent PCT/FR2003/002611 (2003)

[61] Serres, J. et al. "A vision-based autopilot for a miniature air vehicle: joint speed control and lateral obstacle avoidance", Autonomous Robots, 25 (2008), 103-122

[62] Hengstenberg, R. « Das Augenmuskel System der Stubenfliege Musca domestica » Kybernetik 2 (1971) 56-77

[63] Franceschini, N., Chagneux , R., « Repetitive scanning in the fly compound eye » in: Göttingen Neurobiology Rep., N. Elsner, H. Wässle (Eds.), Georg Thieme, Stuttgart (1997) p 279

[64] Mura, F, Franceschini, N. "Obstacle avoidance in a terrestrial mobile robot provided with a scanning retina, in: Intelligent Vehicles II” (Aoki, M., Masaki, I., eds.), (1996) pp 47-52

[65] Viollet, S., Franceschini, N._« Biologically-Inspired Visual Scanning Sensor for Stabilization and Tracking »

IEEE Intern. Conf. Intell. Rob. Syst. (IROS 99) Kyon-gyu, Corea, (1999) pp. 204-209

[66] Viollet, S., Franceschini, N. « Visual servo-system based on a biologically-inspired scanning sensor » in: Sensor fusion and decentralized control, SPIE Vol. 3839, Bellingham, USA, (1999) pp. 144-155

[67] Franceschini, N.; Viollet, S.; Boyron, $M$ «Method and device for hyperacute detect. of an essentially rectilinear contrast edge and system for fine following and fixing of said contrast edge» Patent PCT/FR2005/11536 (2005)

[68] Viollet, S., Michelis, J.,_Franceschini, N. « Toward a bio-inspired nonemissive powerline detection system » In : Proc. 32th European Rotorcraft Forum, ERF, (2006), Maastricht, Paper N AD09

[69] Viollet, S., Franceschini, N. " A high speed gaze control system based on the Vestibulo-Ocular Reflex » Robotics and Autonomous Systems 50 (2005), 147-161

[70] Kerhuel, L., Viollet, S., Franceschini, N. «A sighted aerial robot with fast gaze and heading stabilization » In : IEEE Intern. Conf. on Robots and Systems (IROS 07), San Diego, USA (2007)

[71] Srinivasan, MV. et al.

«Honeybee navigation en route to the goal: visual flight control and odometry » J. Exp. Biol., 199 (1996) 237-244

[72] Srinivasan MV.et al. «How honeybees make grazing landings on flat surfaces » Biol. Cyb. 83 (2000), 171-183

[73] Srinivasan, MV. et al.

"Range perception through apparent image speed in feely flying honeybees" Visual neuroscience, 6 (1991) $519-535$

[74] Kirschfeld, K.; Franceschini, N., Minke, B.

« Evidence for a sensitizing pigment in fly photoreceptors » Nature 269 (1977) 386-390 